\title{
ATIVIDADE MUSCULAR DURANTE A MARCHA APÓS ACIDENTE VASCULAR ENCEFÁLICO
}

\author{
Fernanda Ishida Corrêa, Flávia Soares, Daniel Ventura Andrade, \\ Ricardo Mitsuo Gondo, José Augusto Peres, Antônio Olival Fernandes, \\ João Carlos Ferrari Corrêa
}

\begin{abstract}
RESUMO - Objetivo: Buscar parâmetros da marcha de pacientes após ter sofrido acidente vascular encefálico (AVE) com hemiparesia. Método: Comparados 15 voluntários pós-AVE e 15 voluntários saudáveis com a mesma idade, gênero e peso. A comparação foi feita por eletromiografia utilizando cinco pares de eletrodos de superfície do lado comprometido (espástico) e um eletrogoniômetro sobre o eixo articular de rotação da articulação do tornozelo em estudo. Resultados: O início da atividade eletromiográfica, a partir dafase de apoio, para os músculos glúteo medial, reto femoral, tibial anterior, sóleo, e porção medial dos isquiotibiais foi significantemente ativados anteriormente durante o ciclo da marcha nos voluntários pós-AVE. O final da atividade eletromiográfica para os músculos reto femoral, tibial anterior, sóleo, e porção medial dos isquiotibiais foi significantemente prolongado nos voluntários pós-AVE. Voluntários pós-AVE demonstraram também mais co-ativação dos músculos agonistas e antagonistas da articulação do tornozelo e joelho durante a fase de balanceio. Conclusão: Essas alterações e co-contrações musculares da marcha permitem que os voluntários pós-AVE adotem um padrão de marcha mais seguro e mais estável para compensar a diminuição da informação sensorial da articulação do tornozelo.
\end{abstract}

PALAVRAS-CHAVE: acidente vascular encefálico, eletromiografia, espasticidade.

\begin{abstract}
Muscle activity during gait following stroke
ABSTRACT - Objective: To compare muscle activity and joint moments in the lower extremities during walking between subjects with stroke and control subjects. Method: We compared fifteen healthy volunteers and fifteen stroke patients, with the same age gender and weight data had been compared by electromyography. The system of signals acquisition used consisted of five pairs of electrodes of surface, beyond one electrogoniometer on the axis articulate of rotation of the joint of the ankle in study. Results: Onset times with respect to heel-strike for the medial gluteus, tibialis anterior, soleus, rectus femoris and medial hamstring muscles were significantly earlier during the gait cycle in subjects with stroke than in contro I subjects. The cessation times of soleus, tibialis anterior, rectus femoris, and medial hamstring muscles were significantly prolonged in subjects with stroke. Conclusion: Subjects with stroke showed more co-contractions of agonist and antagonist muscles at the ankle and knee joints during stance phase. These gait changes and co-contractions may allow subjects with stroke to adopt a safer, more stable gait pattern to compensate for diminished sensory information from the ankle.
\end{abstract}

KEY WORDS: stroke, electromyography, spasticity.

O acidente vascular encefálico (AVE) apresenta manifestações clínicas que refletem a localização e extensão da lesão vascular ${ }^{1}$. Lesões no sistema corticoespinal após AVE interferem com as atividades de vida diária, mobilidade e comunicação. Pacientes com seqüelas de AVE demonstram dificuldade em controlar o início do movimento, bem como o contrde motor voluntário ${ }^{2}$. A principal causa desta interferência é a espasticidade, fazendo com que haja acometimento da habilidade do paciente em p roduzir e regular o movimento voluntário. A espasticidade pode acarretar deformidades estáticas; contudo, a espasticidade pode também alterar a angulação articular durante a marcha dinâmica ${ }^{3}$. Evidências que suportam este argumento incluem a velocidade angular reduzida em músculos espásticos durante movimento articular isolado ${ }^{4,5}$.

É importante mensurar o padrão de ativação elétrica muscular devido à relação da espasticidade com a sinergia dos músculos agonistas e antagonis-

Clínica de Fisioterapia, Departamento de Ciências da Saúde, Centro Universitário Nove de Julho - UNINOVE, São Paulo SP, Brasil. Recebido 13 Dezembro 2004, recebido na forma final 30 Maio 2005. Aceito 9 Junho 2005. 
tas durante a marcha, bem como o reflexo de estiramento, podendo, portanto, alterar a biomecânica da marcha destes pacientes ${ }^{6}$.

O objetivo deste estudo é quantificar e comparar parâmetros da atividade muscular e momentos articulares da extremidade inferior durante a marcha entre voluntários saudáveis e voluntários pósAVE usando método de eletromiografia (EMG) associado a eletrogoniometria.

\section{MÉTODO}

Os dados foram coletados de 15 voluntários pós-AVE, e 15 voluntários saudáveis com a mesma idade, gênero e peso. O critério de inclusão utilizado foi que todos os pacientes pós-AVE apresentassem hemiparesia, sem história de doenças cárdio-respiratórias, e que tivessem marcha independente. Já os voluntários saudáveis além de não apresentar história de doenças cárdio-respiratórias deveriam apresentar atividade física constantemente. Os pacientes possuíam $4 \pm 2$ anos de seqüelas pós-AVE, e estavam em tratamento fisioterápico contínuo, 2 a 3 vezes por semana para reabilitação funcional da marcha. Todos os voluntários foram informados do protocolo de estudo e assinaram o Termo de Consentimento respectivo.

O sistema de aquisição de sinais EMG utilizado consistiu de cinco pares de eletrodos de superfície do tipo ativo, bipolar e diferencial, utilizado para captação da atividade elétrica dos músculos. O sinal foi pré-amplificado no eletrodo diferencial com ganho de 10 vezes e razão do modo comum de rejeição igual a $80 \mathrm{~dB}$. Um eletrogoniômetroconstituído de duas hastes plásticas, interligadas por um potenciômetro linear e resistência de 10 $\mathrm{K} \Omega$, sobre o eixo articular de rotação da articulação do tornozelo em estudo, permitiu o registro da variação angular contínua e automática.

Esses dois componentes do sistema de aquisição de sinais foram conectados a um módulo condicionador de sinais, em que os sinais analógicos foram filtrados com filtropassa banda de $10 \mathrm{~Hz}$ a $500 \mathrm{~Hz}$, e amplificados novamente, com ganho de 100 vezes, totalizando portanto um ganho final de 1000 .

Os eletrodos de superfície foram colocados sobre o ponto motor dos músculos glúteo médio, reto femoral, tibial anterior, sóleo e porção média dos isquiotibiais do lado comprometido (espástico), acompanhando o sentido longitudinal das fibras musculares (fixados junto à pele por meio de uma fita adesiva de dupla face, internamente, e outra fita adesiva usada externamente aos eletrodos, proporcionando assim melhor fixação destes). A técnica de localização do ponto motor empregada foi a mesma sugerida por Dainty e Norman?.

Após o tratamento do sinal, um traçado médio das passadas do ciclo completo da marcha foi obtido, sendo representativo da atividade funcional do músculo de cada voluntário. Finalmente, um traçado médio representativo da atividade funcional dos voluntários da amostra foi obtido e submetido à análise estatística comparativa, entre os músculos analisados.

Quanto ao eletrogoniômetro, o sistema foi programado para expressar seus valores de variação da tensão elétrica convertidos, por calibração, em graus de movimento articular, durante o movimento das hastes, e que foi utilizado para medir o movimento de flexo-extensão da articulação do tornozelo durante a march $a^{8}$.

Como sistema de referência, utilizamos as medidas sugeridas pela American Academy of Orthopaedic Surgeons, em que a posição neutra da articulação do tornozelo deve ser igual a $0^{\circ} 7$.

Foi realizado o teste estatístico de correlação cruzada, ponto a ponto, durante todo o ciclo da passada (medida de similaridade entre duas amostragens), a fim de podermos comparar o padrão de variação da forma da curva eletromiográfica e eletrogoniométrica em função do tempo.

O tratamento estatístico foi realizado nos programas Excel (Microsoft) e Matlab v. 6.0 (Mathworks), e o tratamento matemático foi realizado nos programas Matlab v. 6.0 (Mathworks) e Origin v. 6.0 Professional (Microcal Software).

\section{RESULTADOS}

As Figuras 1, 2, 3, 4 e 5 apresentam a caracterização do comportamento do sinal eletromiográ fico com respectivo desvio padrão, bem como a média e o C.V., e a distribuição deste durante todo o ciclo da passada no domínio temporal, para os músculos analisados.

O início da atividade eletromiográfica, a part ir do contato do calcâneo no solo (fase de apoio), para os músculos glúteo medial (Fig 1), reto femoral (Fig 2), tibial anterior (Fig 3), sóleo (Fig 4), e porção medial dos isquiotibiais (Fig 5) foram significantemente $(p<0,05)$ ativados anteriormente durante o ciclo da marcha nos voluntários pós-AVE quando comparados aos voluntários saudáveis.

Já o final da atividade eletromiográfica para os músculos reto femoral (Fig 2), tibial anterior (Fig 3), sóleo (Fig 4), e porção medial dos isquiotibiais (Fig $5)$ foi significantemente $(p<0,05)$ prolongado nos voluntários pós-AVE.

\section{DISCUSSÃO}

Momentos de ativação eletromiográfica diferentes durante a marcha, particula rmente nas articulações do joelho e tornozelo, em pacientes hemiparéticos, como seqüela de AVE explicam-se pela espasticidade do membro afetado.

Essa característica do comportamento motor é consistente entre os achados de nosso estudo e de o u tros autore $s^{9}$. Observamos que a redução da ve- 

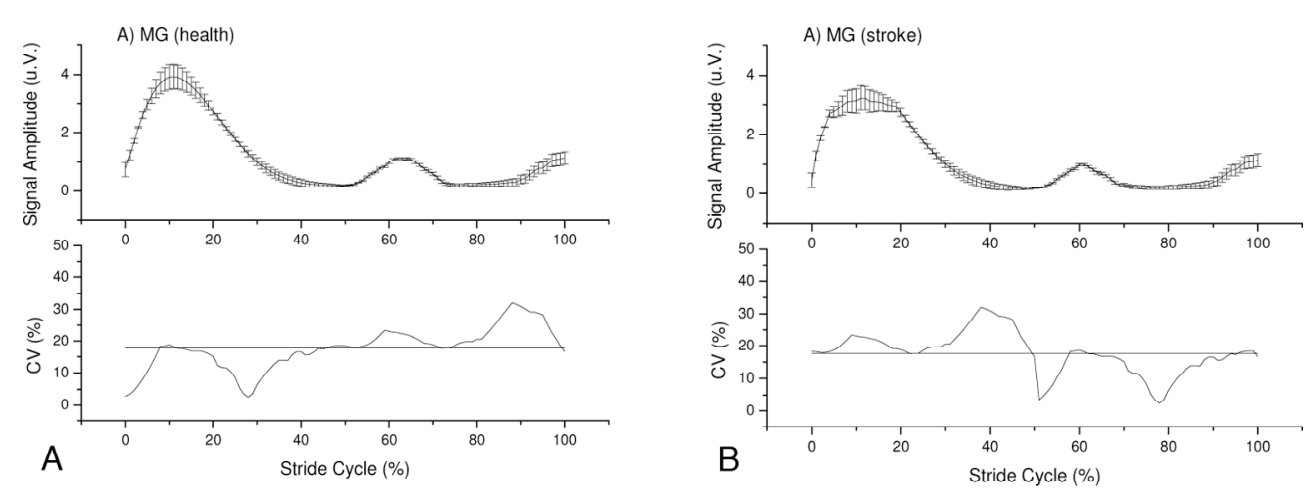

Fig 1. Atividade eletromiográfica do músculo glúteo medial (GM) em voluntários saudáveis e voluntários pósAVE (A e $B$, respectivamente). O gráfico é composto de duas partes: a superior apresenta o comportamento do sinal eletromiográfico inter-sujeitos e respectivo desvio padrão; a inferior apresenta a distribuição dos valores do coeficiente de variação durante o ciclo completo da passada (0-100\%).
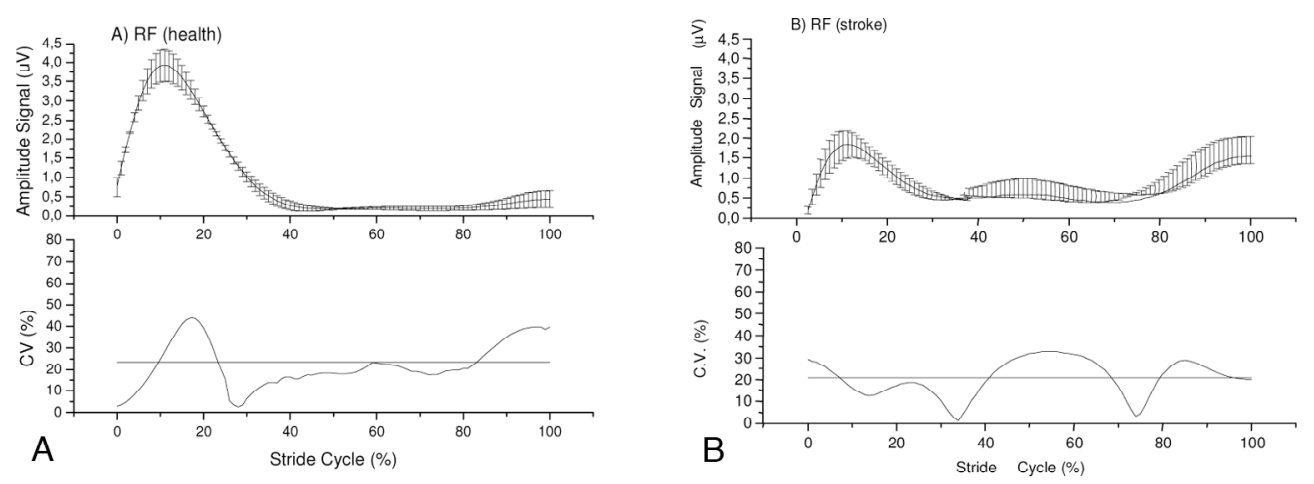

Fig 2. Atividade eletromiográfica do músculo reto femoral (RF) em voluntários saudáveis e voluntários pós-AVE ( $A$ e $B$, respectivamente). O gráfico é composto de duas partes: a superior apresenta o comportamento do sinal eletromiográfico inter-sujeitos e respectivo desvio padrão; a inferior apresenta a distribuição dos valores do coe ficiente de variação durante o ciclo completo da passada (0-100\%).

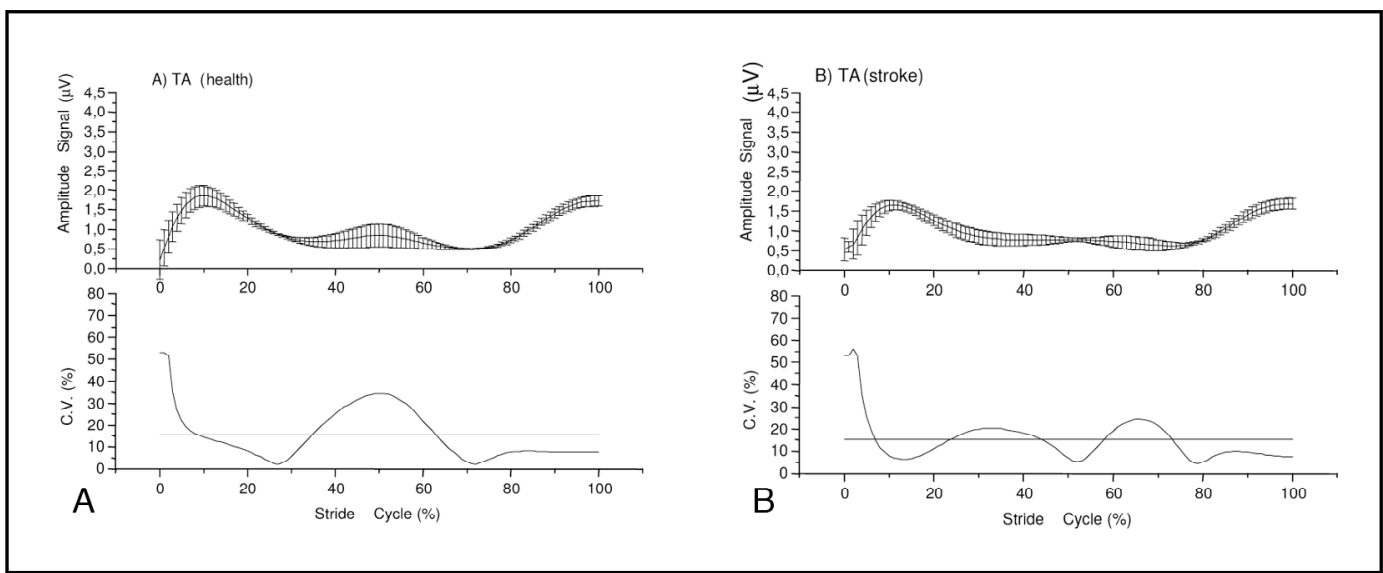

Fig 3. Atividade eletromiográfica do músculo tibial anterior (TA) em voluntários saudáveis e voluntários pósAVE ( $A$ e $B$, respectivamente). O gráfico é composto de duas partes: a superior apresenta o comportamento do sinal eletromiográfico inter-sujeitos e respectivo desvio padrão; a inferior apresenta a distribuição dos valores do coeficiente de variação durante o ciclo completo da passada (0-100\%). 

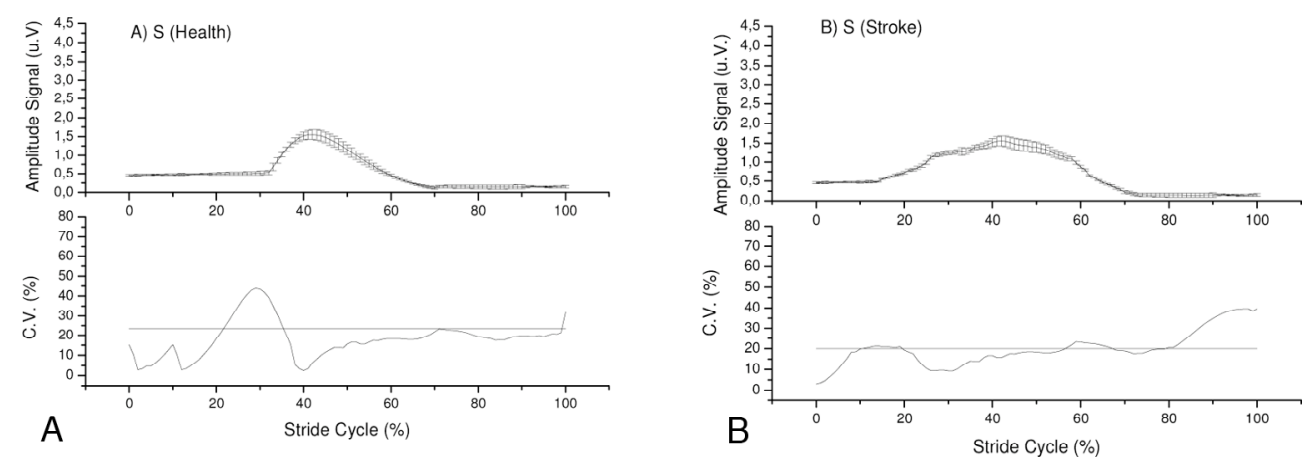

Fig 4. Atividade eletromiográfica do músculo sóleo (S) em voluntários saudáveis e voluntários pós-AVE (A e B, respectivamente). O gráfico é composto de duas partes: a superior apresenta o comportamento do sinal eletro miográfico inter-sujeitos e respectivo desvio padrão; a inferior apresenta a distribuição dos valores do coefi ciente de variação durante o ciclo completo da passada (0-100\%).

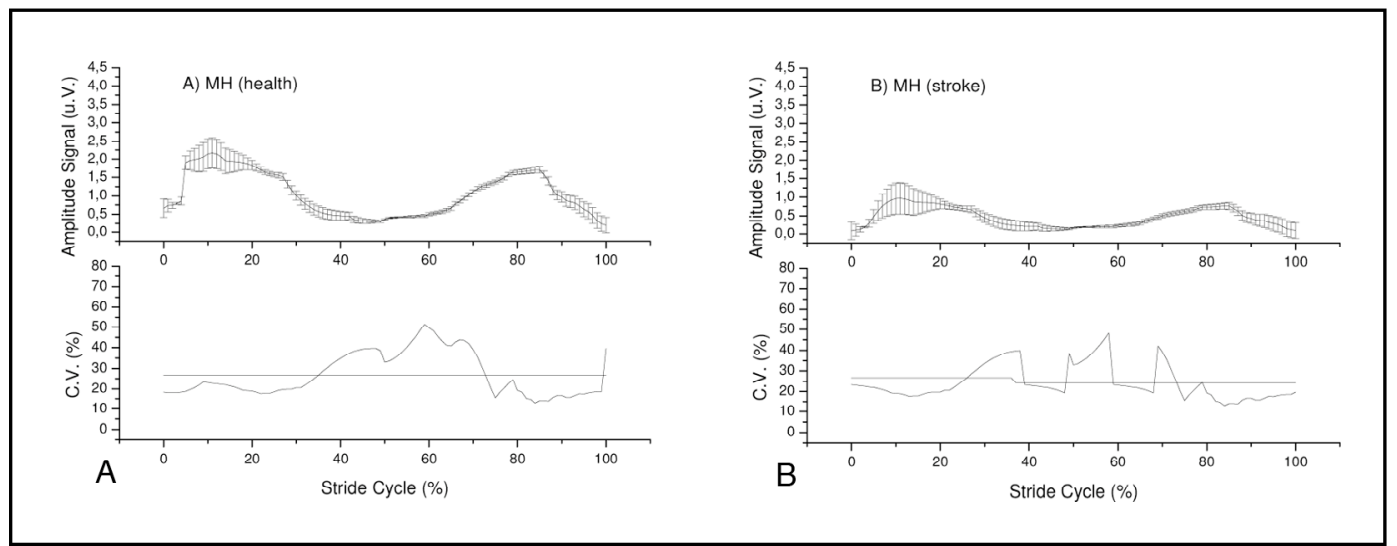

Fig 5. Atividade eletromiográfica da porção medial dos isquiotibiais $(\mathrm{MH})$ em voluntários saudáveis e voluntários pós-AVE ( $A$ e $B$, respectivamente). O gráfico é composto de duas partes: a superior apresenta o comportamento do sinal eletromiográfico inter-sujeitos e respectivo desvio padrão; a inferior apresenta a distribuição dos valores do coeficiente de variação durante o ciclo completo da passada (0-100\%).

locidade angular está diretamente associada com o alto nível de co-atividade muscular em pacientes pós-AVE ${ }^{10}$. Conseqüentemente, velocidade angular pode envolver hipertonia espástica (velocidade-dependente) em músculos antagonistas ou mesmo coespasticidade dos músculos agonistas/antagonistas, gerando forças de desaceleração, limitando, conseqüentemente, a magnitude da velocidade angular ${ }^{11}$.

Esses resultados implicam que os mecanismos neurais da espasticidade reduzam o controle motor durante a marcha, ao contrário, contribuindo inclusive para limitar a excursão articular na marcha espástica. Isso ajuda a explicar porque pacientes hemiparéticos pós-AVE apresentam principalmente um aumento da cadência em lugar de aumentar o comprimento do passo para aumentar a velocidade da marcha.
Atividades de desaceleração (co-ativação) dos músculos flexores do joelho, durante a fase final de balanço, conduz a uma reversão na velocidade articular do joelho além de contribuir para anteversão do quadril na fase de apoio. Co-ativação dos músculos quadriceps/isquiotibiais aumentou nos pacientes pós-AVE. Acreditamos ser essa a causa do atraso da reversão da extensão articular do joelho durante a fase de balanço. Como resultado, a articulação do joelho movimentou-se em direção a uma hiperextensão durante o contato do pé na fase de apoio, impondo cargas de reação anormais no joelho e aumento do gasto de energ $i$ a .

Voluntários pós-AVE demonstraram maior coativação dos músculos agonistas e antagonistas da a rticulação do tornozelo e joelho durante a fase de balanceio quando comparados aos voluntários saudáveis. 
Os resultados demonstram também que os voluntários pós-AVE têm menor mobilidade da articulação do tornozelo, velocidade da marcha diminuída, fase de balanceio prolongada, e menor amplitude da dorsi-flexão da articulação do tomozelo, flexão plantar do tornozelo, e extensão do joelho quando comparados aos voluntários saudáveis.

Em conclusão, estas alterações e co-ativações do padrão da marcha podem permitir que os voluntários pós-AVE adotem um padrão mais seguro e mais estável da marcha para compensar a diminuição das informações sensoriais do tornozelo. A ativação prematura do músculo sóleo e gastro cnêmio nos voluntários pós-AVE pode contribuir à distribuição plantar da pressão plantar anormal. Pesquisa adicional é necessária para esclarecer o relacionamento entre a ativação prematura dos músculos dos triceps surae e os parâmetros da pressão plantar do pé após-AVE.

\section{REFERÊNCIAS}

1. Radanovic M. Características do atendimento de pacientes com acidente vascular cerebral em hospital secundário. A rq Neuropsiquiatr 2000; 58:41-52.

2. Fellows SJ, Kaus C, Thilmann AF. Voluntary movement at the elbow in spastic hemiparesis. Ann Neurol 1994;36:397-407.

3. Knutson E, Martensson A. Dynamic motor capacity in spastic paresis and its relation to prime mover dysfunction, spastic reflexes and antagonist co-activation. Scand J Rehab Med 1980;12:93-106.

4. Corcos DM, Gottlieb GL, Penn RD, Myklebust BM, Agarwal GC. Movement deficits caused by hyperexcitable stretch reflexes in spastic human. Brain 1986;109:1043-1058.

5. Lin JP, Brown JK, Brotherstone R. Assessment of spasticity in hemiplegic cerebral palsy: II. Distal lower-limb reflex excitability and function. Devel Med Child Neurol 1994;36:290:303.

6. Phillips CA, Repperger DW, Chelette TL. The acceleration-velocity relationship: identification of normal and spastic upper extremity movement. Comput Biol Med 1997;27:309-328.

7. Dainty DA, Norman RW. Standarding biomechanical testing in sports. Champaingn: Human Kinetics, 1987.

8. Basmanjian JV, DeLuca CJ. Muscle alive: their functions revealed by electromyography. 5.Ed. Baltimore: Williams \& Wilkins, 1985.

9. Krawetz P, Nance P. Gait analysis of spinal cord injured subjects effects of injury level and spasticity. Arch Phys Med Rehab 1996;77:635-638.

10. Falconer K, Winter DA. Quantitative assessment of co-contraction at the ankle joint in walking. Electromyog Clin Neurophysiol 1985;25:135-149.

11. Abel MF, Damiano DL, Pannunzio M, Bush J. Muscle-tendon surgery in diplegic cerebral palsy: functional and mechanical changes. J Pediat Orthop 1999;19:366-375. 